\title{
Selectivity of perception changes adaptation level
}

\author{
W. LAUTERBACH \\ Institute of Psychology, Düsseldorf University, West Germany
}

\begin{abstract}
In a $2 \times 2$ experimental design, subjects rated blocks of 12 tones (9-point rating scale) preceded by corresponding lights. The two highest tones were aversively conditioned (shock). The experimental group had the opportunity to avoid these tones, the control group did not. Thus the experimental group was able to limit its perceptual experience of the tones, while the control group was not able to do so. In accordance with hypotheses, the self-limitation of the perceptual range affected subjects' perceptions (indicated by stimulus rating and identification). Perceptual change, however, seemed also to affect and increase avoidance behavior; thus stimulus avoidance behavior changed stimulus perception, and changes in stimulus perception resulted in changes of avoidance behavior.
\end{abstract}

The perception, identification, and assessment of a stimulus (tone, weight, hue, etc.) depend not only on its physical characteristics (frequency, etc.), but also on the perceiver's frame of reference and on the position of the stimulus in that frame (Helson, 1964; Thomas, 1974). The frame of reference, in turn, depends on what other stimuli of the same stimulus dimension have previously been perceived, as well as on the ones currently present. It follows that the same stimulus may be perceived differently in different contexts, and that two objectively different stimuli put within different contexts may elicit the same perceptual response.

There are two aspects of all experiments concerned with adaptation level (AL) that are relevant here. First is the fact that a person's frame of reference is usually changed by having him perceive stimuli in addition to those perceived initially. These additional stimuli fall into two categories: (1) more or less extreme stimuli (anchor stimuli) such as were used by Helson (1964) and his co-workers, as well as by Sarris (1968); (2) additional stimuli forming a series from which subjects are asked to select the one that they were first presented (Thomas, 1974).

The second aspect concerns the fact that it is the experimenter who determines what range of stimuli subjects are presented. While this seems to be necessary for the experiment's sake, we should ask whether we may justifiably generalize from such experiments to perceptual processes in nonexperimental situations. We ought to take account of the fact that, in most situations we ourselves determine to some degree what to perceive and what not to perceive. The critical question, then, is whether our frame of reference (or AL) is determined by the range of stimuli we know to exist or the range of stimuli we choose to perceive. As will be pointed out later, this may have implications for the theory of phobic avoidance behavior. In an attempt to answer the

The author is indepted to E. R. Wist, Düsseldorf, for carefully reading the first draft and giving valuable suggestions concerning the clarity and linguistic style of this paper. Requests for reprints are to be sent to W. Lauterbach, Düsseldorf, Institute of Psychology, Düsseldorf University, West Germany. question whether choosing not to perceive can change one's frame of reference, the following hypothesis was formulated: If subjects are given the opportunity to avoid high-pitch tones and are motivated to do so, their ALs will, after a number of avoidance responses, be lower than the frame of reference (or $A L$ ) of subjects who cannot avoid the perception of these tones.

In order to test this hypothesis, the subjects in an experimental group (Group E) could avoid the tones that were associated with shock by pressing a lever before the onset of the tone. They could avoid a tone before hearing it only because tones were preceded by lights whose brightness corresponded to the pitch of the tones. The set of conditions (light signal, lever, and shock) that enabled and motivated subjects to avoid certain tones will henceforth be referred to as the "avoidance condition." The avoidance condition for the subjects of Group C were the same, except for an experimental variation of its consequences; whenever the subjects of the control group (Group C) pressed the lever before the onset of a tone associated with shock, the tone was still presented, but the shock was not administered (see Table 1). Thus the behavior required from subjects of both groups was the same: to press a lever whenever they decided that the next tone might be "dangerous" and thus avoid the shock. The groups differed, however, in that subjects of Group C could then still hear the tone, while subjects of Group E could not.

Table 1

Experimental Design

\begin{tabular}{lll}
\hline & \multicolumn{2}{c}{ Factor A } \\
\cline { 2 - 3 } Factor B & Reduced Stimulus Range & Full Stimulus Range \\
\hline & Group E & Group C \\
Active & Avoidance Condition & Avoidance Condition \\
Selection & Reduced Stimulus Range * & Full Stimulus Range \\
& Group YE & Group YC \\
Passive & No Avoidance Condition & No Avoidance Condition \\
Reception & Reduced Stimulus Range * & Full Stimulus Range \\
\hline
\end{tabular}

*Significant differences 
The introduction of the avoidance condition made it necessary to control for its effect on the perception of the subjects, because in addition to restricting asymmetrically the range of stimuli perceived by the subjects in Group E, it was also possible that these subjects, while choosing not to perceive certain stimuli, would still take them into account, and in this case their perceptual frames of reference might include stimuli not actually perceived. We therefore formulated the hypothesis that the factor "active selection" per se (as opposed to Factor A: reduced stimulus range) has an effect on the stimulus perception, and we tested this hypothesis by introducing a second independent variable: Subjects in Group E were allowed, as mentioned earlier, to avoid a number of high-pitch tones in a set of tones; subjects in a yoked control group (Group YE) were presented with the same set of tones minus the tones avoided by subjects in Group E, and subjects in another control group (Group YC) were presented with the same set of tones as were received by subjects in Group C (see Table 1).

The experimental design, then, was such that the main experimental group was Group E, while the main control was Group C. There were two additional control groups: One was yoked to Group E and called Group YE and another was yoked to Group C and called Group YC. The four groups differed in two ways, which operationalized the two independent variables. First, they differed in that the range of tones to be perceived and assessed by subjects was either constant (in Group C and YC) or asymmetrically variable (in Groups $E$ and $Y E$ ); if the range of tones was asymmetrically variable, higher frequency tones were missing (i.e., avoided by subjects of Group E). Second, they differed in that they either had the avoidance conditions available (Groups $\mathrm{E}$ and $\mathrm{C}$ ) or not (Groups YE and YC). Subjects under the avoidance condition determined themselves which tones to perceive and assess (Group E), while subjects not under the avoidance condition (Group YE) were simply presented with an asymmetrical range of tones, the composition of which was determined by their yoked "partners" under the avoidance condition (i.e., Group E). This difference between Groups E and YE defined the second independent variable: active selection vs. passive reception of stimuli.

\section{METHOD}

\section{Subjects}

A total of 80 paid naive volunteers (university students) served as subjects, 20 in each of the subgroups of the experiment.

\section{Apparatus and Stimuli}

The experimental procedure was controlled by a microprocess computer that produced tones of various frequencies, switched on and off lights with intensities corresponding to the tones, and triggered shocks. There were 12 different tones, $1.5 \mathrm{sec}$ in duration, representing triads of the musical scale to insure subjectively equidistant intervals $(196,262,330,392$, $523,659,784,1046,1318,1568,2093$, and $2637 \mathrm{~Hz}$ ). The tones were preceded by light signals whose brightnesses corresponded to the pitch of the tones; that is, the brighter a light, the higher the associated tone. The lights and tones will be referred to with the numbers 1 to 12 from lowest to highest brightness and pitch. The lights were presented for $1 \mathrm{sec}$ and preceded the tones by $1 \mathrm{sec}$. There was an interval of $5 \mathrm{sec}$ between each tone and the next light signal to give subjects time to assess the pitch of the tone on a 9-point scale (Helson, 1964). Shocks were generated by a shock generator and delivered via two electrodes to the subject's nondominant forearm. Shocks lasted $1 \mathrm{sec}$ and were individually calibrated to be "unpleasant but not too painful."

To avoid a tone (and associated shock) subjects could press a lever located comfortably close to the dominant hand. A 9-point rating scale (cf. Helson, 1964) was located at eye level. Subjects in Groups $\mathrm{E}$ and YE were run simultaneously under yoked conditions. They were located in identically equipped adjacent rooms.

\section{Procedure}

Part 1. After fixing two electrodes to the subjects' forearms and finding a level of shock that was unpleasant but not too painful, the experimenter presented subjects with the lowest and highest pitch tones and instructed them how to use the 9-point scale. Subjects were told that light signals preceded the tones and that brightness was proportional to the pitch of the tone that followed. They were also urged to rate the tones rather than the lights and were then presented and rated a series of 48 lights plus corresponding tones. This series consisted of four blocks of 12 tones each ordered as follows: $9,5,12,7,3,8,1,10,6,11$, 4,2 . Ratings of the last three blocks were later used to calculate AL 1.

Part 2. During this conditioning phase of the experiment, Tones 11 and 12 were classically conditioned using an aversive UCS (shock). To prevent direct associations between light and shock and between shock and rating, lights were not presented and tones were not rated. The order of tones in each of the three blocks presented was as follows: $4,10,8,3,9,11,1,6$, $2,5,12,7$.

Part 3. During the third part of the experiment, subjects could avoid the aversive stimulus (shock). If, after seeing a light signal, they thought that a "dangerous" tone $(11$ or 12$)$ would follow, they could press the lever. In the experimental group (E), pressing the lever had the effect of avoiding both tone and shock. In the control group (C), pressing the lever had the effect of avoiding only the shock.

The block of tones presented in Part 1 was then presented 10 times. AL 2 was calculated from ratings obtained for Blocks 8-10. Subjects in Groups $E$ and $C$ could press the lever before any of the 12 different tones (and in Group $E$ avoided these tones). But if they failed to press the lever before Tones 11 and 12 , they were shocked after hearing the tone.

Part 4. Subjects were then presented with tones in ascending order. They were instructed to identify the tone followed by shock by pressing the lever upon hearing it. The tone that was identified by a subject as being followed by shock was identified as his or her "top tone."

It should be noted that the subjects of the YE and YC groups were instructed to rate the tones they heard on the 9-point scale during Parts 1 and 3. In order to control for differential rating experience during Part 2, subjects of the YE and YC group just listened to the tones without rating them.

\section{RESULTS}

Since intervals between stimuli were subjectively equal, the relationship between stimuli and ratings can be described by means of a linear function, $y=a x+b$. AL was calculated by setting $y=5$ (i.e., the central value of the 9-point scale) and calculating $x$.

The two hypotheses were tested by means of a 
two-way analysis of covariance. An analysis of covariance was used, because the amount of change was expected to (and did) depend also on subjects' initial ALs. The covariate was, therefore, a subject's initial AL (AL 1), while his final AL (AL 2) constituted the variate.

Group changes in ALs before and after stimulus avoidance series are shown in Figure 1, while Table 2 shows the cell means and standard deviations of the covariate and the variate and the adjusted means of the variate.

The analysis of covariance indicated that Factor A (reduced vs. full stimulus range) was highly significant $[F(1,75)=13.76, p<.0001]$, while Factor B (active selection vs. passive reception) was not $[F(1,75)=1.10$, n.s.] ; nor was the interaction between factors $A$ and $B$ statistically significant $[F(1,75)=.08, n . s$.$] . Beta was$ estimated at .435 and shows that subjects' initial and final ALs are in fact not independent; that is subjects with a higher initial AL shifted further than subjects with an initially lower AL. Individual comparisons ( $t$ tests) between the adjusted means showed that Groups $\mathrm{E}$ and $\mathrm{C}$ differed significantly [ $\mathrm{t}(38=2.515$, $\mathrm{p}<.02$, and Groups $\mathrm{YE}$ and $\mathrm{YC}$ also differed significantly $[\mathrm{t}(38)=2.273, \mathrm{p}<.05]$. The remaining individual comparisons between groups were not significant (see Table 1).

In the course of the investigation, the experimenter was under the impression that subjects in the experimental group (E) developed a higher frequency of avoidance responses than did control group (C) subjects. An analysis of covariance was therefore calculated in order to see if this impression was justified. The number of subjects' avoidance responses in the last three series $(8,9,10)$ served as the variate, while the number of subjects' avoidance responses in the first two series ( 1 and 2 ) were the covariate.

Table 3 shows the cell means and standard deviations of the covariate and the variate and the adjusted mean of the variate. The means describe the mean number of avoidance responses of the first two and the last three series. Group E developed a significantly higher rate of avoidance responses than Group C $[F(1,37)=8.57$, $\mathrm{p}<.01\}$.

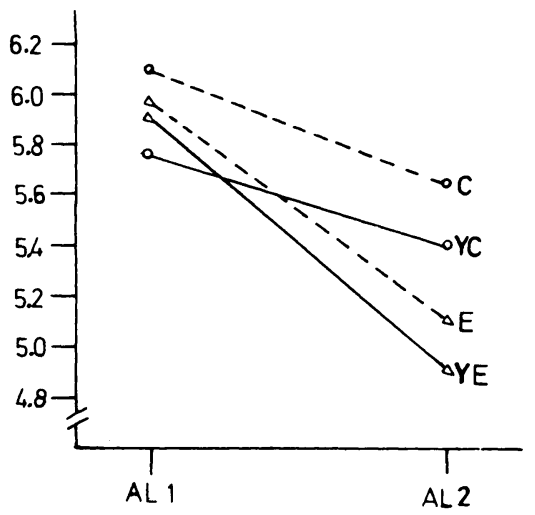

Figure 1. Adaptation Level changes in the four groups. AL 1 = adaptation level in Series 2-4 of Part 1 of the experiment; AL 2 = adaptation level in Series 8-10 of Part 3.
Table 2

Adaptation Level: Means and Standard Deviations of Covariate and Variate and Adjusted Means of Variate of the Four Groups

\begin{tabular}{|c|c|c|c|c|c|}
\hline \multirow[b]{2}{*}{ Group } & \multicolumn{2}{|c|}{ Covariate (AL 1) } & \multicolumn{2}{|c|}{ Variate (AL 2) } & \multirow{2}{*}{$\begin{array}{c}\text { Adjusted } \\
\text { Variate }\end{array}$} \\
\hline & Mean & $\mathrm{SD}$ & Mean & SD & \\
\hline $\mathrm{E}$ & 5.97 & .56 & 5.12 & .62 & 5.11 \\
\hline YE & 5.92 & .86 & 4.92 & .60 & 4.93 \\
\hline C & 6.11 & .52 & 5.65 & .55 & 5.58 \\
\hline YC & 5.77 & .80 & 5.40 & .89 & 5.47 \\
\hline
\end{tabular}

Note $-E=$ experimental, $C=$ control, $Y E=$ yoked to $E, Y C=$ yoked to $C$.

Table 3

Number of Avoidance Responses: Means and Standard Deviations of Covariate and Variate and Adjusted Means of Variate of the Experimental (E) and Control (C) Groups

\begin{tabular}{cccrrr} 
& \multicolumn{2}{c}{ Covariate } & \multicolumn{2}{c}{ Variate } & Adjusted \\
Group & Mean & SD & Mean of \\
E & 4.95 & 1.36 & 10.10 & SD & Variate \\
\hline C & 4.45 & 1.28 & 7.05 & 2.80 & 7.24 \\
\hline
\end{tabular}

The number of avoidance responses for the covariate is based on the first two blocks of tones, while the number for the variate is based on the last three blocks.

In order to analyze (post hoc) the discriminability of the lights and to determine why Group E subjects pressed the lever more often than did control subjects, the average frequency of avoidance responses per tone for all tones was calculated (Table 4). Although subjects in Group $\mathrm{E}$ avoided more tones than did subjects in Group C, they did not differ in the number of shocks received. This was determined by comparing the number of subjects who received up to three shocks during Series 8, 9, and 10 in both groups with the number of subjects receiving more than three shocks by means of a chi-square test; the result $\left[\chi^{2}(1)=1.76\right]$ was far from being significant.

\section{Top Tone}

Subjects in Group C identified tone $\bar{T}=9.76$ as aversively conditioned pitch, and subjects in Group E identified tone $\bar{T}=8.20$ as the highest pitch. The difference was highly reliable $[\mathrm{t}(38)=3.605, \mathrm{p}<.001]$.

\section{DISCUSSION}

Results support the main hypothesis of the experiment; that is, the ALs of subjects who avoided high-pitch tones were after a number of avoidance responses, lower than the ALs of subjects who could not avoid the perception of these tones. The ALs of subjects who could avoid did not, however, differ from the ALs of subjects who heard and assessed the same reduced set of tones without influencing its selection. In other words, a subject's frame of reference appears to be determined by the stimuli he actually perceives, and it changes if he himself chooses not to perceive a number of extreme stimuli.

The results also demonstrate the fact that ALs can be changed not only by adding to the range of stimuli perceived (e.g., by anchor stimuli) but also by reducing it.

Initially, there had been no hypothesis concerning the differential rate of avoidance responses; nevertheless, the differ- 
Table 4

Average Number of Avoidance Responses Per Tone for the Experimental (E) and Control (C) Groups for the First Two (A) and the Last Three (B) Blocks of Avoidance Trials

\begin{tabular}{cccccccccccc}
\hline \multicolumn{1}{c}{ Tones } \\
\hline E $^{1-7}$ & $\mathrm{E}^{8}$ & $\mathrm{C}$ & $\mathrm{E}^{9}$ & $\mathrm{C}$ & $\mathrm{E}^{10}$ & $\mathrm{C}$ & $\mathrm{E}^{11}$ & $\mathrm{C}$ & $\mathrm{E}^{12}$ & $\mathrm{C}$ \\
\hline
\end{tabular}

$\begin{array}{lllllllllllll}\mathrm{A} & 0 & 0 & 1.0 & 1.5 & 4.5 & 4.5 & 13.5 & 12.0 & 15.5 & 12.5 & 15.5 & 14.0\end{array}$

$\begin{array}{rrrrrrrrrrrrr}\text { B } & 0 & 0 & 4.3 & .7 & 9.7 & 4.0 & 15.0 & 8.0 & 18.0 & 15.7 & 19.3 & 19.3\end{array}$

ence between Group $\mathrm{E}$ and $\mathrm{C}$ was significant at the end of the avoidance trials. The question arises as to how to explain this difference. If one assumes that the lights were poorly discriminable (discriminability was not controlled directly), one could argue that Group C subjects had a better chance to improve their light discriminations, for after responding to a light with avoidance, they could still hear the tone corresponding to the light, while Group E subjects could not. If Group C subjects developed a better light discrimination, they would be better able to avoid only the tones associated with shock and thus reduce their overall rates of avoidance responses. ${ }^{1}$ The post hoc analysis shows, however, that (1) discrimination was quite good at the beginning of the avoidance trials (Table 4, line A); (2) both groups learned equally well to better identify the lights signaling the aversively conditioned Tones 11 and 12 ; and (3) it is true that there is a reduction of avoidance responses to Tone 10 in Group $\mathrm{C}$ that may be interpreted as an improved ability to discriminate Light 10 from Light 11 . On the other hand, there is a large increase of avoidance responses from the beginning (A) to the end (B) of the avoidance trials (see Table 4) in Group E, particularly for Tones 8 and 9, but also for Tone 10, which supports the notion that the differential rate of avoidance is mainly due to an increase of avoidance in Group $\mathrm{E}$ rather than to a decrease of avoidance in Group C. Therefore, it cannot be concluded that the lights were poorly discriminable, and discriminability does not account for the differential rate of avoidance responses between the two groups.

The development of a higher rate of avoidance responding over trials by the subjects of Group $\mathrm{E}$ is, at present, not theoretically clear (it would be unreasonable to assume that Group E "unlearned" to discriminate), but it has wider implications that are consistent with the theory that there is a connection between stimulus avoidance and AL shift on the one hand and phobic behavior on the other (Lauterbach, 1979). It is typical of most phobic patients to avoid the stimuli they are afraid of and then to be afraid of and avoid ever more harmless situations. The increase of avoidance responses in Group $E$ parallels the increase of avoidance responses of phobic patients, and both may have to do with AL shifts. Nevertheless, the increase of avoidance behavior cannot be directly explained with AL shifts in the way Lauterbach (1979) explains the increase of phobic behavior, because in this experiment subjects' perceptions (indicated by their ratings and "top tones") changed in one sense modality (pitch of tones), but subjects responded with avoidance to the stimuli of another sense modality (brightness of lights). A model that explains how perceptual change in one modality effects change in another modality associated with the first by classical conditioning, as is the case in this experiment, has not yet been designed and tested.

\section{REFERENCES}

Helson, H. Adaptation level theory. New York: Harper \& Row, 1964.

LAUTERBACH, W. Learning plus: Towards a multifactorial theory of phobias. I. Adaptation level of phobic stimuli. Journal of Behavior Therapy and Experimental Psychiatry, 1979, 10.

SARRIs, V. Absolute or relative anchor effectiveness. Psychonomic Science, 1968, 13, 307-308.

Tномаs, D. $R$. The role of adaptation-level in stimulus generalization. In G. H. Bower (Ed.), The psychology of learning and motivation: Advances in research and theory. New York: Academic Press, 1974.

\section{NOTE}

1. The author is grateful to a reviewer for pointing out that this may be an explanation for the differential rate of avoidance responses and thus stimulating the preparation of Table 4.

(Received for publication January 31 , 1979.) 